\title{
Enhanced Surface Mechanical Properties and Microstructure Evolution of Commercial Pure Titanium Under Electropulsing-Assisted Ultrasonic Surface Rolling Process
}

\author{
Yong-Da Ye ${ }^{1,2} \cdot$ Xiao-Pei $\mathrm{Li}^{1,2} \cdot$ Zhi-Yan Sun $^{1,2} \cdot$ Hai-Bo Wang ${ }^{1} \cdot$ Guo-Yi Tang ${ }^{1}$ \\ Received: 3 January 2018/Revised: 8 February 2018/Published online: 25 April 2018 \\ (C) The Chinese Society for Metals and Springer-Verlag GmbH Germany, part of Springer Nature 2018
}

\begin{abstract}
The effects of electropulsing-assisted ultrasonic surface rolling process on surface mechanical properties and microstructure evolution of commercial pure titanium were investigated. It was found that the surface mechanical properties were significantly enhanced compared to traditional ultrasonic surface rolling process (USRP), leading to smaller surface roughness and smoother morphology with fewer cracks and defects. Moreover, surface strengthened layer was remarkably enhanced with deeper severe plastic deformation layer and higher surface hardness. Remarkable enhancements of surface mechanical properties may be related to the gradient refined microstructure, the enhanced severe plastic deformation layer and the accelerated formation of sub-boundaries and twins induced by coupling effects of USRP and electropulsing. The primary intrinsic reasons for these improvements may be attributed to the thermal and athermal effects caused by electropulsing treatment, which would accelerate dislocation mobility and atom diffusion.
\end{abstract}

Keywords Electropulsing-assisted ultrasonic surface rolling process (EP-USRP) - Commercial pure titanium .

Roughness · Hardness · Microstructure evolution

\section{Introduction}

Material performances are strongly depended on their surfaces, since micro-cracks and defects are usually propagated from there. Surface processing techniques, known as an effective way to strengthen the material surface and keep chemical composition unchanged inside the materials, would significantly enhance material performances with less ductility loss of the whole components. For titanium and its alloy, the poor surface integrity has been the major obstacle for their widespread applications [1]. Thus, further improving the surface properties of titanium and its alloys by surface processing techniques is indispensable.

Available online at http://link.springer.com/journal/40195

Guo-Yi Tang

tanggy@sz.tsinghua.edu.cn

1 Advanced Materials Institute, Graduate School at Shenzhen, Tsinghua University, Shenzhen 518055, China

2 School of Materials Science and Engineering, Tsinghua University, Beijing 100084, China
Ultrasonic surface rolling process (USRP), as a newly surface strengthening technology based on severe plastic deformation (SPD), has attracted considerable attention over decades [2-4]. During USRP, the sample surface is struck by a tungsten carbide ball attached to an ultrasonic device vibrating at high frequency. Thus, excellent microstructure, hardness, surface quality and residual stress are received during the process [5-7]. Nowadays, USRP is widely used to improve the fatigue life, corrosion and wear resistance, since the surface nanocrystallization, hardness, compressive residual stress and surface quality are the main features for these enhancements [8-10]. Huang et al. [11] investigated the fatigue behaviors of AISI 316L stainless steel under gradient nanostructure and found that the fatigue strength was significantly enhanced in both the low- and high-cycling fatigue regimes. $\mathrm{Li}$ et al. [6] studied the tribological properties of Ti-6Al-4V alloy under USRP with different frequencies and loads and proposed that the tribological properties are remarkably enhanced due to the ultra-refined grains. However, material strength enhancement is typically accompanied by loss of ductility. Remarkable work hardening in surface strength layer induced by USRP restricts its further plastic deformation inside the alloy and thus limits its 
strengthened depth [12]. Yang et al. [13] investigated the plastic damages by USRP and indicated that $\sim 0.1 \mathrm{~mm}$ plastic damages depth would be formed after treatment, which would seriously affect its surface roughness and deformation ability. Moreover, Panin et al. [14] proposed that pile-ups with various shapes and sizes would be easily formed while suffered repeated severe impacts by USRP. Therefore, it is hard to achieve a significant enhancement both in surface properties and effective depth.

For decades, electropulsing treatment (EPT), as an instantaneous high-energy input method, has been employed to improve the ductility of metal materials [15-17]. EPT has shown a good application in numerous fields, such as improving plastic deformation ability, promoting recrystallization, cracks healing, phase transformation and so on [18-21]. Gromov et al. [22] found that the fatigue strength was enhanced within a short time and the structure, phase composition and dislocation substructure evolution subjected to EPT might be the features. Ronshan Qin et al. [23] studied the crack healing induced by EPT through thermodynamics and proposed that the heterogeneous temperature rise due to Joule heat might be ideal for crack healing. They also investigated low carbon steel subjected to EPT and found that EPT was able to change the precipitation behavior of carbon atoms and affect the distribution of cementite [20]. Zhu et al. [19] proposed that the deformability of $\mathrm{Ni}_{47} \mathrm{Ti}_{44} \mathrm{Nb}_{9}$ shape memory alloy was remarkably enhanced with less oxidation at a relatively low temperature. However, up to now, studies related to the effect of electropulsing on USRP for Ti alloys are still insufficient.

In the present work, a novel coupling technique called electropulsing-assisted ultrasonic surface rolling process (EP-USRP), which based on high-energy electropulsing and SPD, is carried out to target the shortcomings of USRP for Ti alloys. Wang et al. [12, 24] indicated that EP-USRP had potential advantages over traditional USRP on enhancing the surface mechanical properties and refining grains for AISI 304 stainless steel. However, few studies have investigated the effects of EP-USRP on the enhancements of surface mechanical properties and the operating mechanism of EP-UNSM in commercial pure titanium. Therefore, the effect of EP-USRP on surface mechanical properties, microstructure evolution on commercial pure titanium as well as its mechanism is investigated in this article.

\section{Experimental}

\subsection{Materials and Processing Technique}

The annealed commercial pure titanium bars were investigated as original studied materials. The chemical composition of as used titanium alloy is shown in Table 1. Turning process was performed before USRP and EPUSRP to obtain uniform sample dimensions $(\Phi 14.8 \mathrm{~mm} \times 150 \mathrm{~mm})$ and surface morphology characteristics as much as possible with the following parameters: cutting speed $(25.6 \mathrm{~m} / \mathrm{min})$ and feeding rate $(55 \mathrm{~mm} / \mathrm{min})$.

The USRP and EP-USRP were carried on a self-design platform based on a CNC lathe. The schematic diagram of the EP-USRP process and installation is shown in Fig. 1. An ultrasonic apparatus equipped with a scrollable rolling tip with a hardness of more than 90 HRA, surface roughness of Ra $0.1 \mathrm{~mm}$ and radius of curvature of $6 \mathrm{~mm}$ was employed. During processing, high electropulsing current was conducted through the sample within a short time and combined with ultrasonic treatment to obtain surface strengthened layer. The parameters of USRP are presented in Table 2. Samples are repeatedly processed for three times to obtain uniform SPD layers. The electropulsing current was produced by a self-designed power generator with a sharp waveform, and the parameters of EPT are shown in Table 3. (Root-mean-square current is the mean effective current density during EPT.) The L-G 8 guide rail oil was used in the process for cooling and lubricating.

\subsection{Measuring and Characterizing}

The axial surface roughness of each specimen was evaluated by a Mitutoyo SJ-210 portable surface roughness tester, and the surface morphology was characterized by a

Table 1 Chemical composition of commercial pure titanium (wt $\%$ )

\begin{tabular}{llllll}
\hline $\mathrm{Ti}$ & $\mathrm{H}$ & $\mathrm{O}$ & $\mathrm{Fe}$ & $\mathrm{C}$ & $\mathrm{N}$ \\
\hline Bal. & 0.006 & 0.12 & 0.14 & 0.02 & 0.03 \\
\hline
\end{tabular}

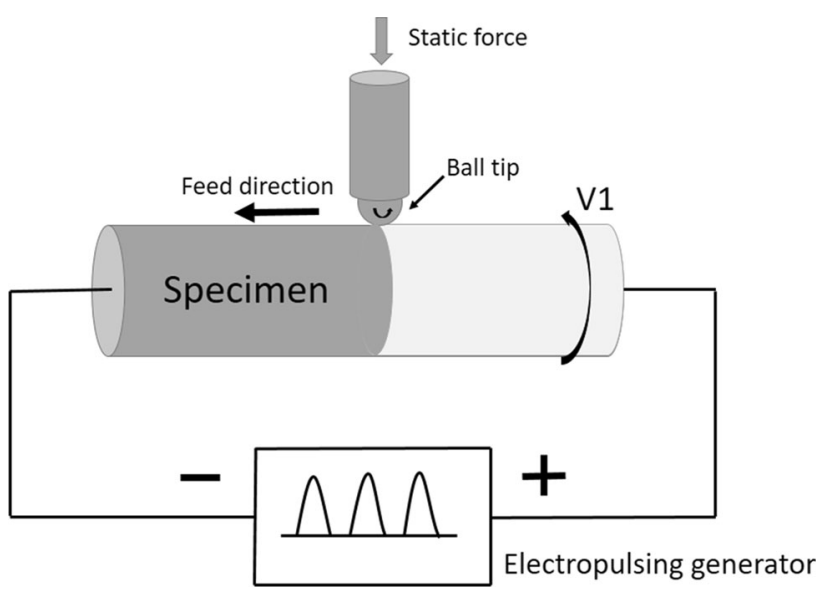

Fig. 1 Schematic diagram of the EP-USRP process and installation [25] 
Table 2 Processing parameters of USRP

\begin{tabular}{lllll}
\hline Rolling line speed $(\mathrm{m} / \mathrm{min})$ & Feeding rate $(\mathrm{mm} / \mathrm{min})$ & Frequency $(\mathrm{kHz})$ & Static force $(\mathrm{N})$ & Amplitude $(\mu \mathrm{m})$ \\
\hline 12.1 & 13 & 27 & 1030 & 6 \\
\hline
\end{tabular}

Table 3 Processing parameters of EPT

\begin{tabular}{lllll}
\hline Frequency $(\mathrm{Hz})$ & Root-mean-square current $\left(\mathrm{A} / \mathrm{mm}^{2}\right)$ & Amplitude current $\left(\mathrm{A} / \mathrm{mm}^{2}\right)$ & Duration $(\mu \mathrm{s})$ & Temperature $\left({ }^{\circ} \mathrm{C}\right)$ \\
\hline 500 & 0.95 & 5.0 & 135 & 104 \\
\hline
\end{tabular}

HIROX KH-7700 three-dimensional optical stereomicroscope. The micro-hardness of strengthened layers was measured by using a HVS-1000B micro-Vickers tester with a $200 \mathrm{~g}$ load and dwell time of $15 \mathrm{~s}$. The nano-hardness was tested by using a Keysight Nano Indenter G200. The microstructure and electron backscatter diffraction (EBSD) analyses were conducted on a Hitachi S4800 scanning electron microscope (SEM).

\section{Results}

\subsection{Surface Roughness and Morphology}

The axial surface roughness and morphology of specimens after USRP and EP-USRP are depicted in Fig. 2. After turning, the mean values of surface roughness are $\sim \mathrm{Ra}$ $0.867 \mu \mathrm{m}$ at steady-state period as shown in Fig. 2a. The surface roughness receives remarkable enhancement when USRP and EP-USRP are applied. However, the one obtained by EP-USRP is more remarkable with a surface roughness value of $\sim \mathrm{Ra} 0.33 \mu \mathrm{m}$ compared to the USRP sample of $\sim \operatorname{Ra} 0.45 \mu \mathrm{m}$. It can be seen that several fluctuations at certain position exist on the curve of USRP sample as presented in Fig. 2b. These fluctuations would seriously restrict the further improvement in surface roughness. Just as depicted in Fig. 2c, these fluctuations can be reduced with smaller surface roughness and more uniform curve by EP-USRP. Carefully comparing the morphology in Fig. 2a'-c', some obvious morphological differences at micro-level can be noticed. After turning process, many turning marks standing with high peaks and valleys appear on the surface. These turning marks can be eliminated to some degree by USRP. However, some micro-cracks still exist on the surface. Undoubtedly, these micro-cracks would seriously limit its performance, since cracks would initiate and propagate from here and result in serious influence on fatigue life of components. It can be found that these potential negative effects can be significantly reduced when EP-USRP is applied. After EP-USRP, the surface is smoother with few micro-cracks and defects, which may give reasons for the less fluctuations and lower values of the surface roughness curve after EP-USRP. This phenomenon shows that EP-USRP can facilitate microcracks healing and reduce defects to further improve the surface quality.

\subsection{Surface Hardness}

The cross-sectional micro-hardness distributions within the strengthened layer and nano-hardness at the depth of $50 \mu \mathrm{m}$ induced by USRP and EP-USRP are characterized in Fig. 3. For the turning specimen, the micro-hardness is $\sim 215 \mathrm{HV}$ at the top surface and kept almost constant with increasing distance to the surface. As shown in Fig. 3a, after treatment, both USRP and EP-USRP receive improvement in the surface maximum micro-hardness and the strengthened layer depth. For USRP, the micro-hardness at $40 \mu \mathrm{m}$ depth increases from the initial state of $\sim 215$ to $\sim 257 \mathrm{HV}$, which indicates an increased rate of $19.5 \%$. Then, the micro-hardness begins to decrease sharply as the depth increases, and finally levels off $\sim 380$ $\mu \mathrm{m}$. However, the EP-USRP-induced specimen shows the more remarkable enhancement in surface maximum microhardness and strengthened layer depth with the value of $\sim 284 \mathrm{HV}$ (increased rate of 32.1\%) and $\sim 550 \mu \mathrm{m}$, respectively. Similar situations can be observed from Fig. 3b, which shows the nano-hardness at $50 \mu \mathrm{m}$ depth below the surface. It can be observed that EP-USRP would produce more pronounced nano-hardness than traditional USRP. For turning sample, the mean value of nano-hardness is $\sim 3.15 \mathrm{GPa}$. Then, USRP is carried out for materials, the mean value of nano-hardness increases to $\sim 3.75 \mathrm{GPa}$, which indicated a strengthening rate of $\sim 19 \%$. The nano-hardness will get remarkable enhancement when EP-USRP is applied, which prepares a mean nano-hardness over 3.9 GPa with a strengthening rate of $\sim 24 \%$. Therefore, it can be seen that the EP-USRP can not only improve the surface hardness but also improve the depth of the strengthened layer, which may contribute to the enhancement of surface mechanical properties. 

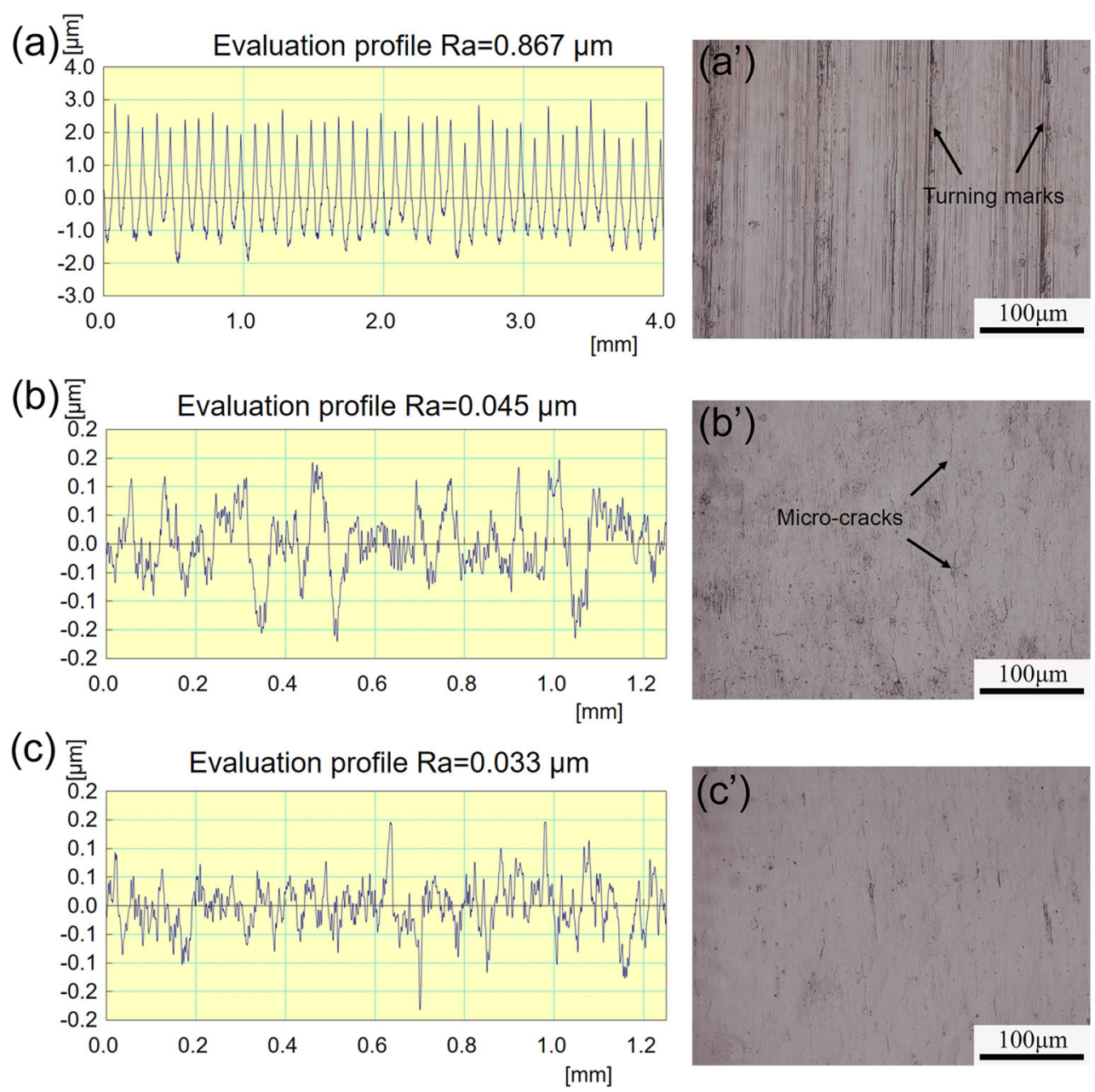

Fig. 2 Axial surface roughness and morphology of specimens after USRP and EP-USRP: a and a' turning; b and b' USRP; c and c' EP-USRP

(a)

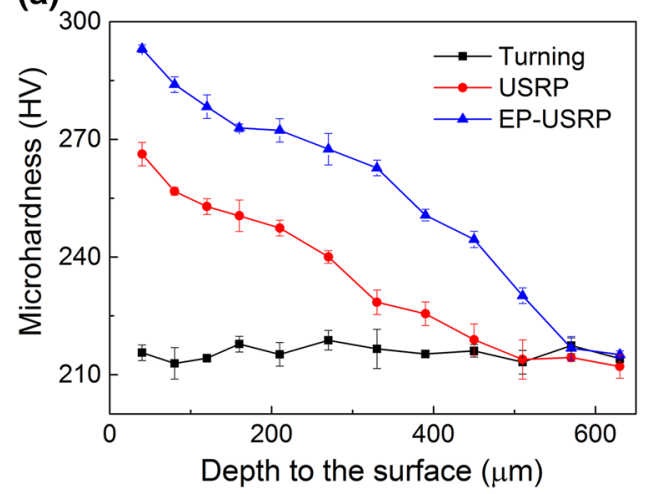

(b)

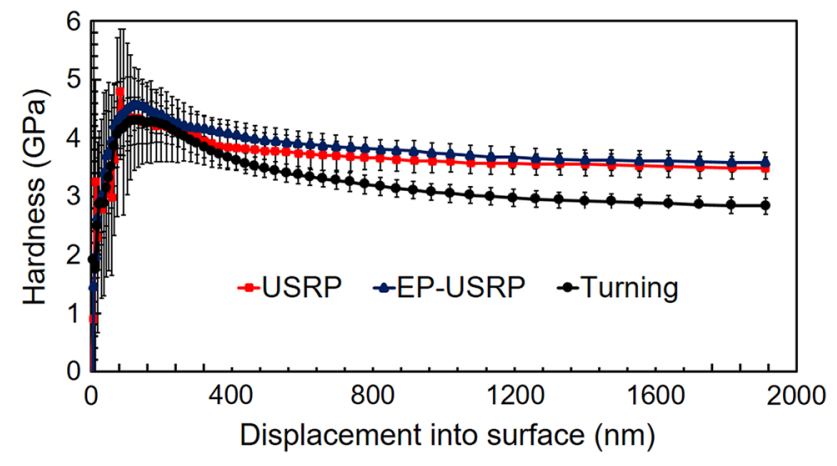

Fig. 3 Cross-sectional micro-hardness distribution within the strengthened layer: a nano-hardness at the depth of $50 \mu \mathrm{m}$; $\mathbf{b}$ induced by USRP and EP-USRP

\subsection{Surface Strengthened Layer}

The microstructure of strengthened layer induced by USRP and EP-USRP is illustrated in Fig. 4. Figure 4a presents the turning microstructure, which shows a large amount of grains located irregularly with random orientation. Then, USRP is applied on the specimens, a gradient microstructure layer with a depth of about $200 \mu \mathrm{m}$ lies on the top of the surface, and grains are deformed into strips one by one. It is obvious to see that a more considerable SPD layer was 

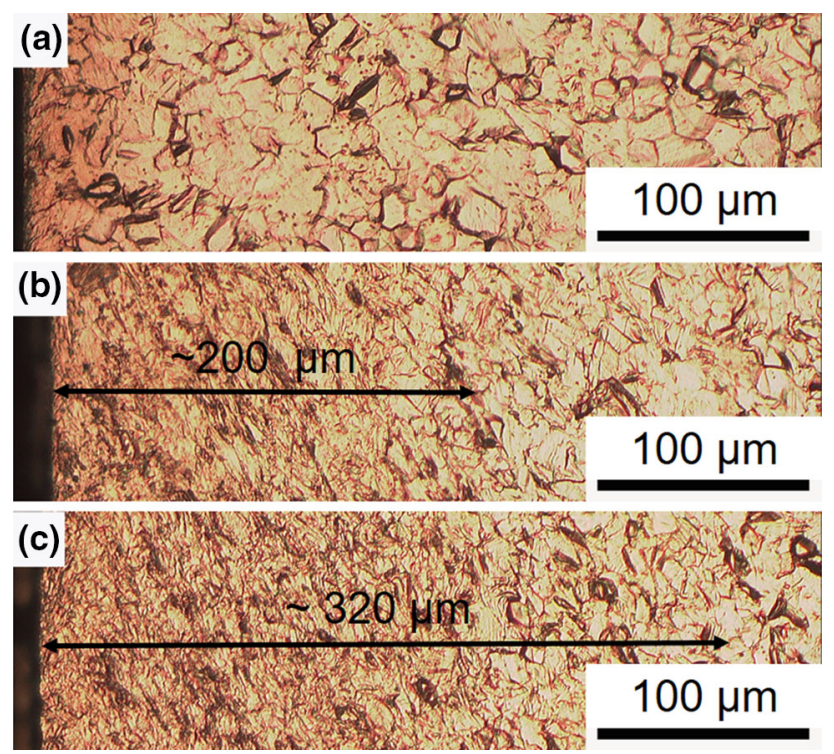

Fig. 4 Microstructure of strengthened layer induced by USRP and EP-USRP: a turning; b USRP; c EP-USRP

obtained by EP-USRP with a strengthened layer depth of over $320 \mu \mathrm{m}$ as characterized in Fig. 4c. In addition, the degree of plastic deformation on the surface microstructure is more severe than that of USRP specimen with grains elongated in the direction paralleled to the sample surface. Furthermore, it is hard to identify the grain boundaries in the range of $0-150 \mu \mathrm{m}$ depth, since the deformation degree is much more severe than USRP sample. Thus, EP-USRP would produce deeper and more pronounced SPD layer, leading to excellent surface properties.

\section{Discussion}

\subsection{Significant Improvement in Strengthened Layer}

It is well known that significant improvements in the surface mechanical properties are strongly attributed to the strengthened SPD layer. For conventional USRP, SPD is brought into the surface layer due to the serious extrusion on the surface by high-frequency collision of rolling tip. During the processing, the time interval between two shocks is so short that the elastic recovery is restricted. Thus, overlap of multi-elastic deformation leads to the plastic compressive deformation even if the stress value is less than the yield strength. At this time, hardness and grains refinements are significantly enhanced during the repeated striking. However, during the severe striking process, the remarkable hardening in surface strengthened layer induced by SPD shows an extreme obstruct for the further plastic deformation [1]. Lu et al. [26] proposed that the severe plastic deformation would cause the introduction of large amounts of immobile dislocations and boundaries, which would restrict the further grain refinement. Just as the micro-hardness shown in Fig. 3, the USRP-induced hardening would cease when reaching a certain depth. In this case, electropulsing is carried out to target the shortcoming of USRP due to the enhancement of plastic deformation ability. Zhu et al. [19, 27] proposed that the ductility of material would obtain significant enhancement due to the acceleration of dynamic recrystallization by electropulsing. Owing to the improvement in deformation ability by EPT, the effect of USRP would be remarkably amplified. That is to say, when EPT and USRP are applied on sample simultaneously, the electropulsing induced better deformation ability which would play an important role by promoting the mobility of immobile dislocations and boundaries. Thus, repeating electropulsing softening and ultrasonic hardening would occur during the EP-USRP processing until reaching its work hardening limitation. Therefore, ultrasonic vibration energy can permeate deeper than USRP process and force dislocation multiplication and annihilation into dislocation walls and tangles as well as subsequent formation of sub-boundaries and boundaries, leading to higher hardness as well as the formation of deeper SPD layers, which can be briefly explained by the microstructure evolution in SPD layer at top surface and at $50 \mu \mathrm{m}$ depth below the surface induced by USRP and EPUSRP as shown in Fig. 5. Figure 5a, a' presents the turning microstructure, the bulk grains stand irregularly with random orientation, and the grain size reaches $\sim 20 \mu \mathrm{m}$. Then, USRP is applied on sample, and grains suffered severe plastic deformation as shown in Fig. 5b, b'. Some grains are deformed into strips. However, some unchanged grains can still be observed. The materials suffered more severe plastic deformation as EP-USRP is carried out with all of the grains being deformed into strips. By carefully comparing the microstructure at $50 \mu \mathrm{m}$ below the surface as illustrated in Fig. 5a'-c', the deformation degree is gradually enhanced by the application of USRP and the most severe strengthened layer is finally obtained by EPUSRP. For the EP-USRP sample, the grains are parallel to each other and the gaps of each parallel strip are too close to identify, which indicated an excellent strengthened layers. Thus, EP-USRP has a significant improvement in surface strengthened layer, which may be attributed to the excellent deformation ability from dynamic recrystallization by EPT.

\subsection{Evolution of Microstructure}

The observed surface mechanical enhancements are mainly attributed to the ultra-refined grains through dynamic recrystallization. Jiang et al. [28] indicated that 


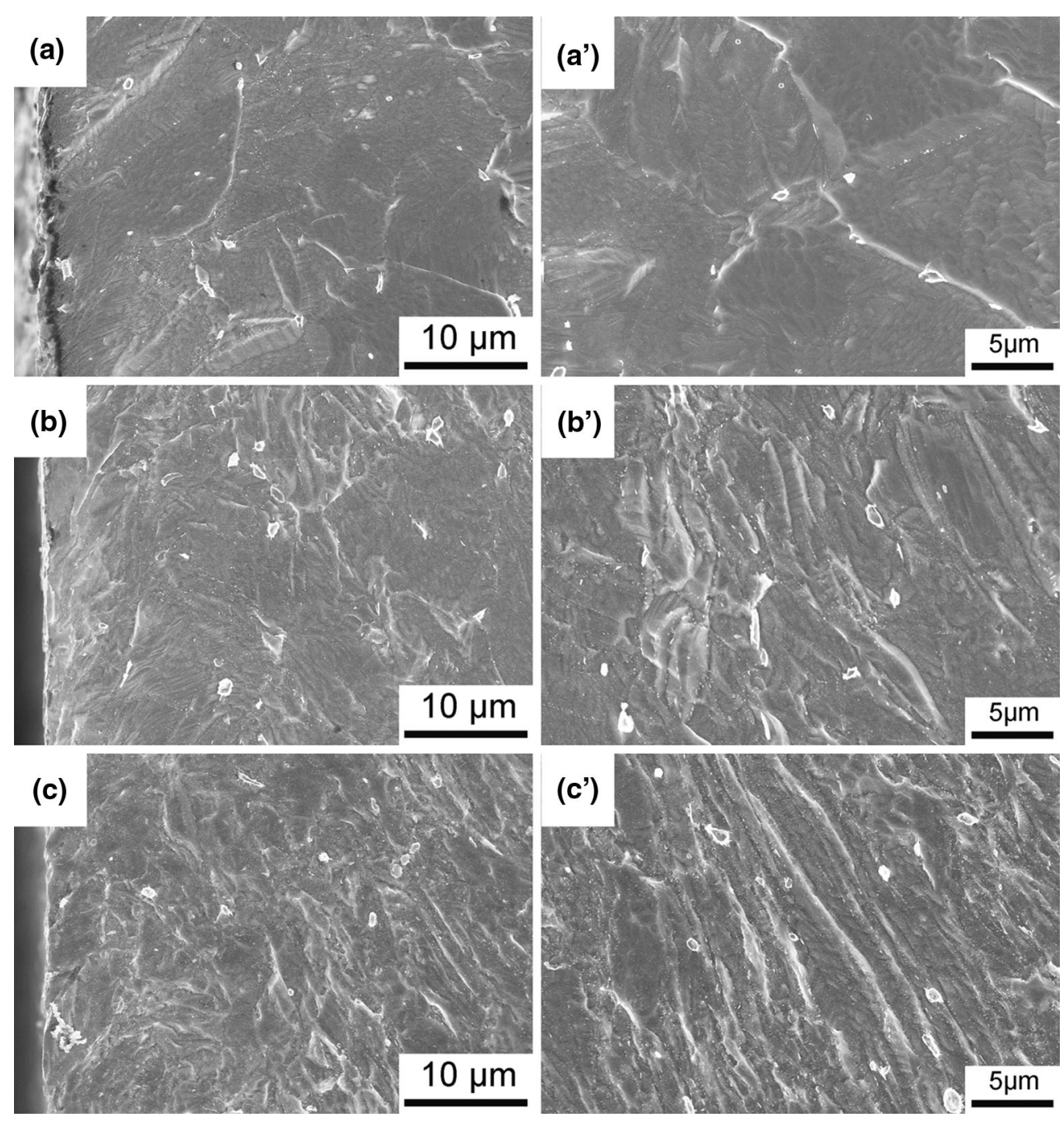

Fig. 5 Microstructure of plastic deformation layer at top surface and $50 \mu \mathrm{m}$ below the surface induced by USRP and EP-USRP: a turning at the surface; a' turning at $50 \mu \mathrm{m}$ below the surface; b USRP at the surface; b' USRP at $50 \mu \mathrm{m}$ below the surface; c EP-USRP at the surface; c' EPUSRP at $50 \mu \mathrm{m}$ below the surface

recrystallization mainly involved formation and growth of recrystallization nuclei and the nucleation rate strongly depended on the incubation period. In general, the incubation period is considered as the total time required for rearrangement of dislocation substructure, formation of sub-grain and generation of high angle boundary with high mobility, which depended on the mobility of dislocation. For plastic deformation, SPD layers can be obtained through serious extrusion on the surface by high-frequency collision of the rolling tip. Serious distorted grains have very high dislocation density and energy storage. When the density of dislocations reaches a yield value, dislocation cells and dislocation tangles are originated by the crossslippage of many dislocations accompanied by the increased strain extent $[5,29,30]$. Then, with the accumulation of dislocations in the subsequent plastic deformation, dislocation cells and dislocation tangles turn into sub-boundary and finally into grain boundary. With increasing grain boundary, these boundaries would turn into nucleation area, leading to grain refinement through the dynamic recrystallization. The EBSD maps at $250 \mu \mathrm{m}$ below the surface are shown in Fig. 6. It can be observed that EP-USRP has an advantage over conventional USRP on refining grains. After the USRP, some bulk grains are divided into small fragments near the $250 \mu \mathrm{m}$ depth as presented in Fig. 6a. The formation of so many subboundaries indicates excellent refinement here. However, further increasing the depth to the surface, the grain refinement becomes weaker and weaker. Especially at $\sim 500 \mu \mathrm{m}$ depth, the grains lie unchanged. It is obvious to see that the grain refinement is much more outstanding than USRP sample in EP-USRP sample with more refined grains as shown in Fig. 6b. Moreover, by carefully comparing the EBSD maps, more sub-boundaries are formed 

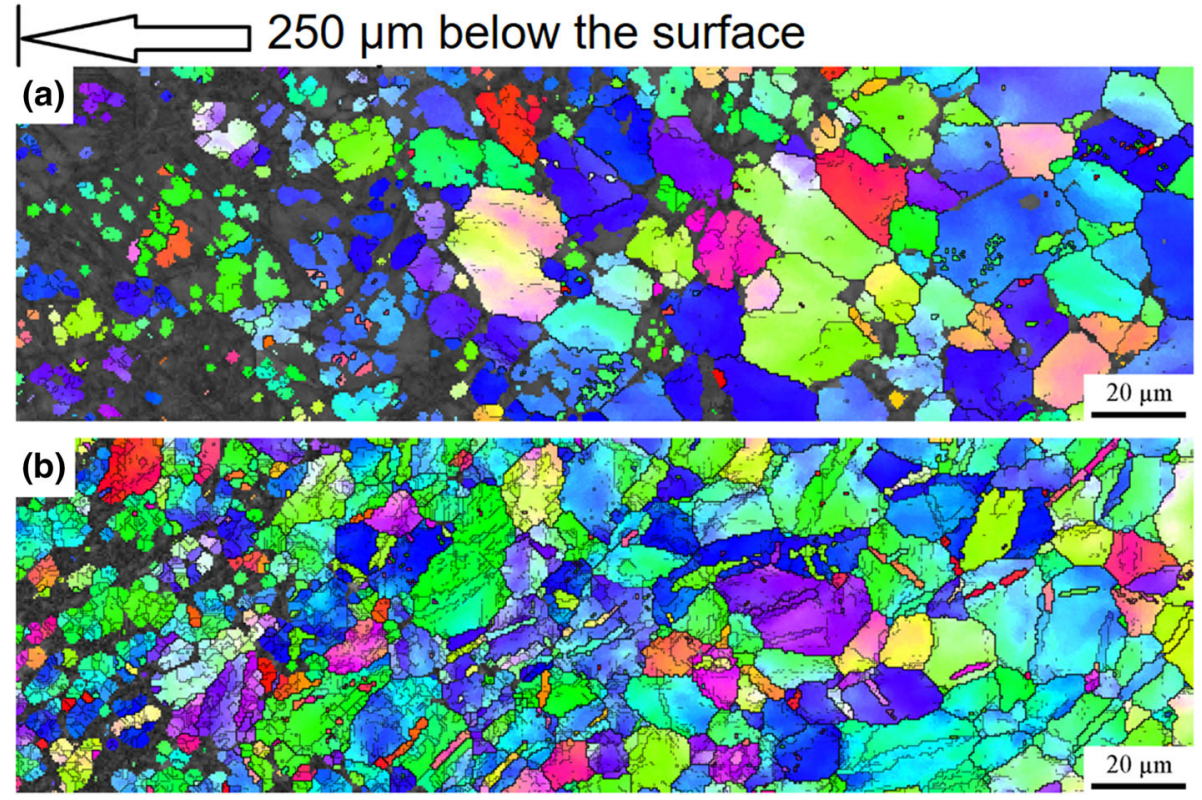

Fig. 6 EBSD maps at $250 \mu \mathrm{m}$ depth below the surface induced by a USRP; $\mathbf{b}$ EP-USRP

by EP-USRP, which indicates a more severe plastic deformation and dynamic recrystallization. When EPUSRP is applied on metals, both thermal and athermal effects exerted by electropulsing would promote dislocation mobility and atom diffusion positively. The thermal effect is similar to high-rate heating called Joule heat, which is displayed with the temperature rise [31]. However, during the EP-USRP, the effect of thermal effect is relatively small and may play an assistant role due to the non-directional heat transfer, the relative low temperature ( $\sim 104{ }^{\circ} \mathrm{C}$ and is much smaller than conventional recrystallization temperature of $\mathrm{Ti}$ ) and the limit of energy input. In the athermal effect aspect, as commonly accepted by researchers, it would generate electron wind and do the direct influence on dislocation mobility, atom diffusion and annihilation of point defects [32, 33]. Xu et al. [34] proposed that the athermal effect would promote the diffusion of vacancy, which is tied with the movement of dislocation. Namely, under EPT, nucleation would happen more easily than traditional process at a relative lower temperature (seen in Fig. 6). According to the dynamics, the slipping velocity of the dislocations is determined by the applied mechanical stress. Worked by electropulsing, an additional mechanical stress $F_{\mathrm{e}}$ on unit length of the dislocation by the drift electrons in the same direction is [35]

$F_{\mathrm{e}}=\left(\frac{\Delta^{2} b V_{\mathrm{d}}}{4 V}\right)\left(\frac{V_{\mathrm{e}}}{V_{\mathrm{d}}}-1\right)\left(\frac{\partial n_{0}}{\partial \mu}\right)$,

where $b$ is Burgers vector, $V_{\mathrm{e}}$ is the drift velocity of the electrons, $V_{\mathrm{d}}$ is the speed of dislocations, $V$ is the speed of the electrons in the Fermi plane, $n_{0}$ is the density of the free carrier, $\mu$ is the chemical potential and $\Delta$ is the deformation potential constant. It can be found from Eq. (1) that when $V_{\mathrm{e}}>V_{\mathrm{d}}$, the force of free electrons is normal, i.e., the movement of the dislocations is accelerated. The improvement in the plastic deformation is more obvious with the increase in the current density, which leads to the acceleration of dislocations as well as nucleation of recrystallization.

To sum up, two mechanisms can be related to the accelerated dynamic recrystallization process by EPUSRP. One is the individual recrystallization process by EPT or USRP. As discussed above, the formation and growth of recrystallization nuclei would be promoted due to the acceleration of dislocation mobility by electropulsing or plastic deformation. In general, the ultra-refined grains obtained by the first mechanism would soon turn into subsequent dynamic recrystallization by the repeating impacts. Another and the most important mechanism is the coupling effects of both EPT and USRP. During the process, the materials are more inclined to plastic deforming, which would amplify the USRP effect since EPT would significantly improve the deformation ability of materials. In this case, the plastic deformation is more severe and grains are further refined, from which more pronounced grain-boundary compatibility arose due to the promotion of dislocation accumulation, movement and annihilation during dynamic recrystallization.

It is well known that twins would play a great role during the plastic deformation in titanium and its alloy $[14,29]$. Twins are also known to be the primary deformation mechanism of titanium and its alloys subjected to 
impact loading, since twin systems are necessary to maintain the deformation compatibility $[36,37]$. Wen et al. [30] indicated that twinning could not accommodate a huge amount of deformation due to the fact that the atomic displacement by twin is less than one inter-atomic distance. Thus, twins appear only in the layer in which the strain is low. That is to say, when the stains reach a small level during deformation, twins would appear to maintain the necessary deformation capability. With increasing strains, twins would vanish while reaching a critical level. The distribution of twins induced by USRP and EP-USRP from 400 to $500 \mu \mathrm{m}$ below the surface is characterized in Fig. 7 . Only a few twins exist after USRP, and each of the twin boundaries are very short, which may due to the relatively low strains. The quantities of twin boundaries would receive significant improvement when EP-USRP is carried out for materials. Lots of twins appear in the strengthened layer. These twins are so long that most of them are formed through the whole grain, which indicate a higher strain than USRP at this depth. It should be noted that, unlike subgrain boundaries formed due to excessive dislocation density, the deformation twins in the grains of the surface layer of the titanium specimens are unable to be strong obstacles for the motion of dislocations into the bulk [14]. Thus, during this section, sub-grain boundaries and twins
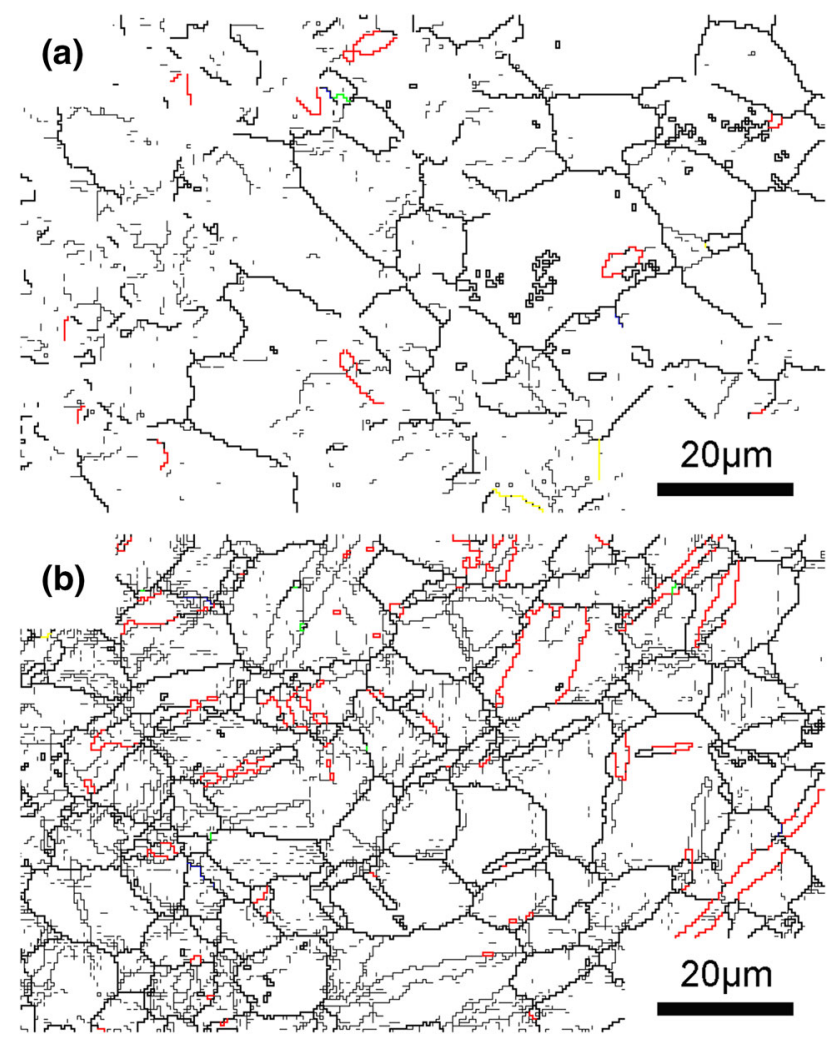

Fig. 7 Distribution of twins induced by a USRP; b EP-USRP at 400-500 $\mu \mathrm{m}$ depth below the surface are formed simultaneously. Since EP-USRP would facilitate the deformation ability of materials, twins would be accumulated during the repeated plastic deformation induced by severe striking. Accordingly, as more plastic strains accumulate, deformation twins in different directions divide the grains into smaller intersections parts and thus generate some rhombic blocks, which eventually lead to refined grains [38]. Moreover, with accumulation of twin boundaries, they would combine with dislocation bands and finally turn into sub-grain boundaries and grain boundaries (seen in Figs. 6, 7). That is to say, twins would promote the mobility of boundaries and thus accelerate recrystallization nucleation rate, contributing to the grain refinement under dynamic recrystallization, and finally result in perfect properties. When electropulsing is applied, the more plastic strains accumulated by electropulsing effect, the more intersection twins will form just as shown in Fig. 7b. Thus, grains would be divided into smaller sections, resulting in excellent grains refinement as well as outstanding properties. Therefore, compared to traditional USRP, EP-USRP would be a significant processing technique to promote excellent dynamic recrystallization.

\section{Conclusion}

Compared to conventional USRP, EP-USRP can remarkably improve the surface mechanical properties and microstructure evolution of commercial pure titanium, leading to smaller surface roughness (from $\sim \mathrm{Ra}$ $0.867 \mu \mathrm{m}$ by turning to $\sim \mathrm{Ra} 0.045 \mu \mathrm{m}$ by USRP and $\sim$ Ra $0.033 \mu \mathrm{m}$ by EP-USRP) and smoother morphology with few cracks and defects. Moreover, surface strengthened layer and ultra-refined grains are significantly enhanced with more severe deformation degree, greater surface hardness (maximum hardness increased rate of $32.1 \%$ by EP-USRP in comparison with USRP of $19.5 \%$ ) and deeper strengthened depth. These enhancements may be attributed to the thermal and athermal effects caused by electropulsing treatment, which would accelerate dislocation mobility and atom diffusion. Thus, more sub-boundaries and twins would be formed through repeating electropulsing softening and ultrasonic hardening during EP-USRP, resulting in excellent grain refinement and mechanical properties. Therefore, EP-USRP is a highly efficient approach to prepare excellent mechanical properties and ultra-refine grains of commercial pure titanium.

Acknowledgements The authors acknowledge the financial support from the Shenzhen Development and Reform Commission Engineering Laboratory Project (Shenzhen development and Reform 2015-1033), the Shenzhen Science and Technology supporting Plan Project (GJHS20160331183313435) and the China Postdoctoral Science Foundation (No. 2017M620770). 


\section{References}

[1] H. Wang, G. Song, G. Tang, J. Alloys Compd. 681, 146 (2016)

[2] C. Ye, A. Telang, A.S. Gill, S. Suslov, Y. Idell, Z. Kai, J.M.K. Wiezorek, Z. Zhou, D. Qian, S.R. Mannava, Mater. Sci. Eng. A 11-12, 613 (2014)

[3] A. Amanov, I.S. Cho, D.E. Kim, Y.S. Pyun, Surf. Coat. Technol. 21, 207 (2012)

[4] B. Wu, P. Wang, Y.S. Pyoun, J. Zhang, R.I. Murakami, Surf. Coat. Technol. 12, 213 (2012)

[5] X.J. Cao, Y.S. Pyoun, R. Murakami, Appl. Surf. Sci. 21, 256 (2010)

[6] G. Li, S.G. Qu, Y.X. Pan, X.Q. Li, Appl. Surf. Sci. 389, 324 (2016)

[7] T. Liu, H. Wang, G. Tang, G. Song, Mater. Sci. Technol. 12, 33 (2017)

[8] A. Amanov, J.H. Kim, Y.S. Pyun, T. Hirayama, M. Hino, Wear 332-333, 891 (2015)

[9] A. Amanov, Y.S. Pyun, S. Sasaki, Tribol. Int. 4, 72 (2014)

[10] A. Amanov, I.S. Cho, Y.S. Pyun, Appl. Surf. Sci. 388, 185 (2016)

[11] H. Huang, Z. Wang, J. Lu, K. Lu, Acta Mater. 87, 150 (2015)

[12] H. Wang, G. Song, G. Tang, Mater. Sci. Eng. A 662, 456 (2016)

[13] X. Yang, J. Zhou, X. Ling, Mater. Des. 36, 477 (2012)

[14] A.V. Panin, M.S. Kazachenok, A.I. Kozelskaya, R.R. Hairullin, E.A. Sinyakova, Mater. Sci. Eng. A 647, 43 (2015)

[15] Y. Jiang, G. Tang, C. Shek, Y. Zhu, Z. Xu, Acta Mater. 16, 57 (2009)

[16] J. Kuang, X. Du, X. Li, Y. Yang, A.A. Luo, G. Tang, Scr. Mater. 114, 151 (2016)

[17] X. Li, G. Tang, J. Kuang, X. Li, J. Zhu, Mater. Sci. Eng. A 9, $612(2014)$

[18] Y. Jiang, G. Tang, C. Shek, Y. Zhu, Appl. Phys. A 3, 97 (2009)

[19] R. Zhu, G. Tang, S. Shi, M. Fu, J. Mater. Process. Technol. 1, $213(2013)$
[20] R.S. Qin, A. Rahnama, W.J. Lu, X.F. Zhang, B. Elliottbowman, Mater. Sci. Technol. Lond. 9, 30 (2014)

[21] Q. Xu, L. Guan, Y. Jiang, G. Tang, S. Wang, Mater. Lett. 9, 64 (2010)

[22] V.E. Gromov, Y.F. Ivanov, V.V. Sizov, S.V. Vorob'Ev, S.V. Konovalov, J. Surf. Investig. X-Ray Synchrotron Neutron Tech. 1, 7 (2013)

[23] R. Qin, S. Su, J. Mater. Res. 8, 17 (2002)

[24] H. Wang, G. Song, G. Tang, Surf. Coat. Technol. 282, 149 (2015)

[25] Y. Ye, H. Wang, G. Tang, G. Song, J. Mater. Eng. Perform., ed. by R. Asthana (2018 in press)

[26] X. Liu, H. Zhang, K. Lu, Science 6156, 342 (2013)

[27] R. Zhu, G. Tang, S. Shi, M. Fu, Mater. Des. 44, 606 (2013)

[28] Y. Jiang, G. Tang, C. Shek, J. Xie, Z. Xu, Z. Zhang, J. Alloys Compd. 536, 94 (2012)

[29] N.R. Tao, Z.B. Wang, W.P. Tong, M.L. Sui, J. Lu, K. Lu, Acta Mater. 18, 50 (2002)

[30] M. Wen, G. Liu, J.F. Gu, W.M. Guan, J. Lu, Appl. Surf. Sci. 12, 255 (2009)

[31] Q. Xu, G. Tang, Y. Jiang, Mater. Sci. Eng. A 13-14, 528 (2011)

[32] Y.H. Zhu, S. To, W.B. Lee, X.M. Liu, Y.B. Jiang, G.Y. Tang, Mater. Sci. Eng. A 1, 501 (2009)

[33] R.F. Zhu, G.Y. Tang, S.Q. Shi, M.W. Fu, V.E. Gromov, Appl. Phys. A 4, 111 (2013)

[34] Z. Xu, G. Tang, S. Tian, F. Ding, H. Tian, J. Mater. Process. Technol. 1, 182 (2007)

[35] G. Tang, J. Zhang, M. Zheng, J. Zhang, W. Fang, Q. Li, Mater. Sci. Eng. A 1-2, 281 (2000)

[36] Y.G. Liu, M.Q. Li, Mater. Lett. 185, 488 (2016)

[37] S. Nemat-Nasser, W.G. Guo, J.Y. Cheng, Acta Mater. 13, 47 (1999)

[38] K.Y. Zhu, A. Vassel, F. Brisset, K. Lu, J. Lu, Acta Mater. 14, 52 (2004) 